\title{
FORTALEZAS Y VILLAS DEL REY: NOTAS SOBRE LA FRONTERA GALAICO-PORTUGUESA EN TIERRAS ORENSANAS A FINES DEL SIGLO XIII
}

\author{
Por \\ CÉSAR OLIVERA SERRANO
}

\begin{abstract}
RESUMEN
Estudio de las fortalezas y villas de la frontera galaico-portuguesa, dentro de la actual provincia de Orense, bajo la inciativa de Alfonso X y Sancho IV de Castilla, a fines del siglo XIII y comienzos del XIV, en la antigua Vía de la Plata, la principal ruta de comunicación terrestre del sur de Galicia con el reino de León. Las fortalezas y pueblas tienen en común la terminación del nombre, en la que se alude claramente a su condición de realengo.
\end{abstract}

\section{PALABRAS CLAVE}

Fortalezas medievales, frontera, repoblación, nobleza, realengo, ruta de la plata.

\begin{abstract}
The work studies a group of towns and fortresses near the frontier between Galicia and Portugal, actually province of Orense, built under
\end{abstract}

«CUADERNOS DE ESTUDIOS GALLEGOS», Tomo XLVIII, Fascículo 114, Santiago 2001. 
the rule of kings Alfonso X and Sancho IV, in the last years of the XIIIth Century and the first years of XIVth Century. These fortresses defended also the old «Rout of Silver», the most important way between the south of Galicia and the kingdom of León. The fortresses' names, ended always with the word «rey» (= «king»), remind their royal origins.

\section{KEYWORDS}

Medieval fortresses, frontier, repopulation, nobility, lauds of the crown, rout of silver.

\section{INTRODUCCIÓN}

Al sur de la provincia de Orense y muy cerca de la raya de Portugal encontramos un grupo de villas y fortalezas que tienen rasgos idénticos en el nombre, o si se prefiere, una filiación clara con el realengo: Viladerrey, Canderrey, Monterrey y Flor de Rey. La primera de la serie fue, como indica su nombre, una villa o puebla real que se encuentra enclavada en el mismo puerto de las Estivadas, en la carretera que comunica el valle de Monterrey con la llanura de La Limia. En cuanto a la segunda, la fortaleza de Canderrey, hoy desaparecida por completo, cabe decir que se encontraba en las cercanías de Viladerrey, probablemente en el alto denominado O Castelo, dominando las alturas del mismo puerto ya citado. La «puebla» y fortaleza de Monterrey se alza encima del antiguo castro de Verín, de Varoncelli o Baruncelle, nombre antiguo que se utilizó hasta el siglo XIII y, por último, la «puebla» de Flor de Rey, en el actual término municipal de Vilardevós, lugar bastante separado de la carretera nacional que circula en las proximidades de La Gudiña, en pleno paso de las tierras orensanas a las de Zamora.

\footnotetext{
${ }^{1}$ Este artículo se ha realizado dentro del proyecto de investigación del Instituto de Estudios Gallegos «Padre Sarmiento», titulado "Inventario gráfico y documental de las fortalezas medievales de Galicia: la provincia de Orense», cuyo investigador principal es D. Eduardo Pardo de Guevara y Valdés.
}

«CUADERNOS DE ESTUDIOS GALLEGOS», Tomo XLVIII, Fascículo 114, Santiago 2001. 
Además del nombre o apellido común (- de Rey), estos enclaves compartieron algo digno de mención: su alineamiento en torno a la antigua Vía de la Plata, a modo de eslabones defensivos que, de paso, custodiaban la línea fronteriza con Portugal. No parece que tantos puntos en común obedezcan al azar, sino más bien a una iniciativa colonizadora de la monarquía en la segunda mitad del siglo XIII, especialmente bajo Alfonso $\mathrm{X}$ y Sancho IV, una época dominada por las tensiones luso-castellanas por la frontera que culminó en la firma del tratado de Alcañices de 1297, durante la minoría de Fernando IV.

No estaríamos aquí, por tanto, dentro de la labor repobladora que la monarquía leonesa hizo bajo Fernando II y Alfonso IX de zonas costeras e interiores de Galicia, que en su día explicó y resumió el profesor Salvador de Moxó ${ }^{2}$, sino más bien ante la fortificación de la frontera con Portugal que en parte ya puso de manifiesto el profesor Julio González en su estudio sobre Alfonso IX, aunque sólo lo hizo en relación con el caso de Verín ${ }^{3}$. Por consiguiente, las circunstancias que explican el desarrollo de estas villas y fortalezas se entienden mejor si se tiene en cuenta la complicada situación luso-castellana anterior y posterior al tratado de Alcañices ${ }^{4}$. De ser cierta esta iniciativa, tendría que contemplarse en relación con las tareas de definición fronteriza que llevaron al otro lado de la raya monarcas como Alfonso III en relación con Chaves, a la que dota de murallas en $1253^{5}$, y también Dinís I ${ }^{6}$ con su conocida política de fortificación en todo el perímetro fronterizo de Portugal.

Las villas y fortalezas «del rey» no fueron ni mucho menos las únicas del realengo orensano de la segunda mitad del siglo XIII, pero estuvieron

\footnotetext{
${ }^{2}$ MOXO, S. de, Repoblación y sociedad en la España cristiana medieval, Madrid, 1979 , pp. 280-282.

${ }^{3}$ GONZÁLEZ, J., Alfonso IX, I, Madrid, 1944, p. 249.

${ }^{4}$ Vid. algunas ponencias del congreso sobre el tratado de Alcañices; BAQUERO MORENO, H., «As relações de fronteira no século de Alcañices (1250-1350): a tratado de Alcañices», IV Jornadas Luso-Espanholas de História Medieval. As relações de fronteira no século de Alcanices, I, Porto, 1998, pp. 641-653. LADERO QUESADA, M.A., «Reconquista y definiciones de frontera», IV Jornadas Luso-Espanholas, I, pp. 654-691.

${ }^{5}$ VENTURA, L., «A Fronteira Luso-Castelhana na Idade Media», IV Jornadas LusoEspanholas, I, pp. 25-52.

${ }^{6}$ BARROCA, M.J., «D. Dinis e a arquitectura militar portuguesa», IV Jornadas LusoEspanholas, I, pp. 801-824.
} 
en un sector geográfico muy disputado en torno a la raya de Portugal y de ahí deriva el interés por conocer un poco mejor sus crcunstancias históricas y, en especial, las épocas en que aparecieron o edificaron, o los tenientes que las custodiaron en nombre del rey, además del ulterior destino que llegaron a tener todas ellas.

Queda todavía bastante por hacer en este campo de las fortalezas en zonas de frontera, pero los datos que han ido saliendo al hilo de la investigación son lo bastante explícitos como para advertir una coherencia política en las iniciativas de los monarcas a los que hemos hecho alusión. Las villas y fortalezas del rey formaron una cierta unidad bajo el mando de algunos vasallos reales, como por ejemplo Pay Méndez de Canderrey, o de grandes personajes como Fernando de Castro, que saldrán a lo largo de las páginas siguientes. Todos los indicios apuntan a que el primero fue el responsable de la denominación común de estos enclaves o, en todo caso, el responsable de unos puntos defensivos que vinieron a llamarse de un modo semejante merced a la iniciativa regia que hizo posible su despegue.

Expondremos la información de cada villa o fortaleza a la luz de la documentación disponible hasta este momento, señalando de manera breve los rasgos más característicos de cada caso. Por desgracia, no quedan hoy día restos visibles de estas fortalezas, a excepción de Monterrey, cuyas espléndidas construcciones datan, por cierto, de épocas muy posteriores a las aquí tratadas. Pero a pesar de esa ausencia física importa mucho conocer la localización de unos puntos defensivos que, por otra parte, se situaron deliberadamente en antiguos castros, lo cual complica bastante las cosas a la hora de datar los yacimientos; falta aún la labor de prospección arqueológica que, probablemente, resolverá bastantes dudas que hoy siguen pendientes de solución.

\section{CANDERREY Y VILADERREY}

La primera mención toponímica de Canderrey data del año 1037, aunque no se habla de un castillo, sino de un castro, "Castro Kenderedi», próximo a una "villa Kenderedi»" ${ }^{7}$, probablemente Viladerrey; la referen-

${ }^{7}$ ANDRADE CERNADAS, J., O Tumbo de Celanova, Santiago, 1995, doc. $\mathrm{n}^{\circ} 268$. 
cia es demasiado escueta para saber si ya existía en ese momento algún tipo de fortificación.

A comienzos del siglo XII encontramos la primera alusión explícita que habla de la existencia de una fortaleza o castillo: se trata de una donación otorgada el 10 de octubre de 1100 por Onega Fernández a favor del abad Pedro de Celanova, por la que le entrega su heredad de «Marci», en Limia, delimitada por diversos lugares, entre los que destaca el «castello Kenderedi»:

«[dono $]$ hereditate mea propria quam habeo de avorum et parentum meorum in Limia, vocabulo verbo dicent Marci, subtus castello Kenderedi, vocabulo ecclesie Sancto Iohanne, et determinat per Sautelo et Trasmires, et de alia parte per Causo et idem per Scornoboves» ${ }^{8}$.

Algunos topónimos que aparecen en este documento, como Sautelo, Trasmires y Scornaboves (actuales Soutelo, Trasmiras y Escornabois) permiten indentificar con bastante exactitud el castello Kenderedi, que se correspondería con el castillo de Canderrey, en al alto denominado hoy día de O Castelo, en Viladerrey.

Un documento de finales del siglo XII nos informa de tres cosas importantes; primero, que la denominación es ya la de Canderrey o Candrei; que, además, era efectivamente un castillo, no un simple castro; y que formaba parte del realengo: el 8 de diciembre de 1198, desde Palencia, Alfonso IX de León entrega a la reina Berenguela 30 castillos de Asturias, León y Galicia, algunos de la comarca de Limia, entre los que está el de Candrei:

«[...]Et si in aliquo castellorum istorum uilla uel habitatores fuerint, ipsi habitatores et moradores eiusdem castelli faciant hominium illi militi qui illud tenuerit de regina domna Berengaria quod sint uasalli fidelis regine domne Berengaria et omnes directos suos ei in pace persoluant, scilicet in Gallecia Sanctum Pelagium de Lodo, Aguilar de Mola, Alba de Bunel [sic, por Buval], Candrei, Aguilar de Pedraio [...]»

${ }^{8}$ ANDRADE, J., Tumbo de Celanova, doc. $\mathrm{n}^{\circ} 284$

${ }^{9}$ GONZÁLEZ, J., Alfonso IX, II, Madrid, 1944, doc. $\mathrm{n}^{\circ} 135$. 
El documento también informa de los tenientes responsables de su custodia:

«[...] Istorum XXX castellorum tenet [...] Pelagius Subredina Sactum Pelagium de Lodo, Aguilar de Mola, Candrei; scilicet, Gonsaluus Iohannis Aguilar de Pedraio; scilicet, Osorius Iohannis Albam de Bueuel $[\ldots] »$

La fortaleza de Canderrey es el primer caso de denominación alusiva al realengo y vuelve a ser citada dentro de un conjunto de fortalezas reales del sur de Galicia en otra dote matrimonial: se trata de un documento del mismo monarca leonés, Alfonso IX, otorgado el 6 de junio de 1217 desde Castel Rodrigo, a favor de sus hijas Sancha y Dulce, donde ahora se emplea, por cierto, el vocablo «castra»:

«[...] do, concedo et confirmo filiabus meis donne Santie te donne Dulcie ista castra, scilicet, Cabreiram de Baroncelli, Lobarcanam, Canderei, Portelam de Santio Iohannis, Riberam, Celme, Asperelo, Araugio, Sanctam Crucem, Burgum de Ripa Avie, Sanctum Iohannem de la Barra, Alleriz, Milmandam et quantum aliud ad me pertinet in tota Limia, exceptis Aguilar de Moa et Aguilar de Pedraio. Do preterea Cruniam cum turri de faro et cum suo alfoz, et Saniurgium, Travam, Morgadanes, Sanctum Martinum de Ladrones... Preterea concedo et confirmo quod si mater earum regina donna Theresa premortua eis fuerit, castra que ipsa tener, scilicet, Entenza, Soveroso, Sanctaenia et Tevura, ad eas devolvantur in tota vida sua...»10.

Trece años má tarde, el 11 de diciembre de 1230, desde Benavente, el nuevo rey, Fernando III, llega a un acuerdo con sus hermanas Sancha y Dulce; por el que les asigna la tenencia en régimen de fieldad de ciertas fortalezas, que en Galicia ascienden a un total de siete, para que así puedan percibir una série de ingresos; las fortalezas gallegas son Lobarcana (Lobarzana), Cabrera (¿Cabreiroá?), Candrex (Canderrey), Allariz, Santa Cruz, San

${ }^{10}$ GONZÁLEZ, J., Alfonso IX, II, doc. $\mathrm{n}^{\circ} 342$. Documento citado por CID RUMBAO, A., Historia de Allariz: villa y corte románica, Ourense, 1984, p. 46.

«CUADERNOS DE ESTUDIOS GALLEGOS», Tomo XLVIII, Fascículo 114, Santiago 2001. 
Juan de Pena Corneira y Milmanda ${ }^{11}$. El rey consigna en este mismo documento de 1230 los nombres de los fieles que tendrán esos castillos:

«Rodericus Gomeç, Ferrandus Iohannis, Pelagius Arie, Iohannes Petri, Arias Ferrandi de Meyra, Petrus Manion, Petrus Marino, Rodericus Pelagii de Candrei et filius eius, filii Melendi Blasqueç, Rodericus Suareç, Martinus Egidii de Asturiis, Sebastianus Gutterrii, Ordonius Alvari et Fernandus Alvari et Pelagius Petri».

El nombre de «Rodericus Pelagii de Candrei», en tanto que fiel o tenente de la fortaleza de Canderrey, aparece citado en ún documento algo anterior, fechado en Castrelo de Veiga el 23 de mayo de 1228, por el que Alfonso IX de León confirma al obispo de Orense sus derechos y privilegios: entre los confirmantes aparece «Pelagius sub redina tenens Candrei et Sanctam Eniam» ${ }^{12}$.

De los datos anteriores se deduce que desde fines del siglo XII Canderrey fue una fortaleza de realengo y probablemente por eso mismo su denominación cristalizó en la forma definitiva. Conservó además esa condición a lo largo del siglo XIII, hasta que en el reinado de Sancho IV (1284-1295) se adjudicó a Pay Méndez de Canderrey.

Los libros de rentas del monarca de los años 1290 y 1292 nos confirman todo lo anterior. En las cuentas de 1290 se dice que el «castiello de Canderey, con la tierra e con los derechos dende» $\rangle^{13}$, que hasta ese momento había tenido asignados en concepto de «tierra» Estéban Fernández, pasaban a ser ahora de Pay Mendez de Canderey, en virtud de una carta expedida en Burgos el 8 de marzo de 1290, por la que el monarca le asignaba a éste último un juro de 100 maravedíes en las rentas y derechos de la feligresía de «San Martín de Vamdes» (sic, por Abavides), en la tierra de Canderrey. En la Colección Salazar y Castro se conserva una copia de este último documento ${ }^{14}$, aunque con fecha de 1289 , no de 1290:

${ }^{11}$ GONZÁLEZ, J., Reinado y diplomas de Fernando III, vol. II, Córdoba, 1983, $\mathrm{n}^{\mathrm{o}} 270$.

${ }^{12}$ Documentos del Archivo de la Catedral de Orense, Orense, 1923; v. I, p. 82-85.

${ }^{.13}$ HERNÁNDEZ, F.J., Las rentas del rey. I, Madrid, 1993, p. 284.

${ }_{14}^{14}$ R.A.H., Col. Salazar y Castro, Índice, M-4 (signatura actual, 9/811), fol. 135v. Reg. CUARTERO Y HUERTA, Índice de la Colección Salazar y Castro, no 45.472.

«CUADERNOS DE ESTUDIOS GALLEGOS», Tomo XLVIII, Fascículo 114, Santiago 2001. 
«[...] por hacer bien y merced a Pay Mendez de Canderey nuestro vasallo, y por servicio que nos fizo y faze damosle el nuestro señorio que nos havemos y devemos haver en la fortaleza de S. Martino de Avavides y cotamosle esta feligresia sobredicha que le non entre y alcaide ni merino ni juez ni otro que ande por nos para fazer y justizia ni la faga sino el o aquellos que él y pusiere, salvo si se ficieren y mal fechos e se cogieren y malfechores y él no quisiere facer en ellos justicia que lo fronten aquellos que oviere por nos a fazer la justicia, y si él no la quisiere facer desde que le frontaren, que entren y facerla. E por hi entrar a facerla una begada que lo non tomen por uso ni entren y otra vegada por esta razon fasta que lo fronten por notario cada vegada que acaesciere. E por le facer mas bien y mas merced damosle todos derechos que solian haver desta fortaleza aquellos que tuvieron la tierra de Canderey por nos, fasta en quantia de cien maravedis de la moneda de la guerra y si mas vale y lo tenemos la para nos. $Y$ todas estas cosas sobredichas le damos que lo aya por juro de heredad para sienpre jamas él e sus fijos e sus nietos e quantos dél vinieren que lo suio ovieren de heredar para vender e dar y enagenar».

La fortaleza de Canderrey y la tierra de Abavides quedaron unidas hasta el siglo XVI como elementos de una sola unidad. Lo interesante del caso es que en Abavides el rey tenía otra fortaleza que, como veremos pronto, también será encomendada a Pay Méndez de Canderrey.

Las cuentas de Sancho IV de 1292 completan los datos anteriores ${ }^{15}$ y añaden que los 1.800 maravedíes anuales que le pertenecían al rey del "castiello de Canderey, con la tierra e con los derechos dende», quedaban puestos a favor de Pay Mendez de Candrey.

Este Pay Méndez de Candrey o Canderrey, sin duda un teniente o alcaide, será premiado en 1295 con otra donación regia, la feligresía de San Salvador de Río Frejo ${ }^{16}$ en Sarreaus; merced que fue confirmada al año siguiente durante la minoría regia de Fernando $\mathrm{IV}^{17}$, de modo que al co-

\footnotetext{
${ }^{15}$ HERNÁNDEZ, F.J., Las rentas del rey, I, p. 285.

${ }^{16}$ R.A.H., Col. Salazar y Castro, M-4 (signatura actual, 9/811), fol. 135v. Reg. CUARTERO Y HUERTA, Índice, $\mathrm{n}^{\circ}$ 45.475, pp. 371-372.

${ }^{17}$ Ibid.
}

«CUADERNOS DE ESTUDIOS GALLEGOS», Tomo XLVIII, Fascículo 114, Santiago 2001. 
menzar el siglo XIV, Pay Méndez de Candrey poseía un pequeño señorío en las tierras de La Limia (Canderrey, Abavides, Sarreaus) conseguido gracias a la generosidad de Sancho IV y Fernando IV. La corona aún conservaba por entonces algunos derechos en Canderrey que finalmente entregó a otras personas: el 20 de marzo de 1303, desde Toledo, Fernando IV hacía donación a Lope López de Ribera, su vasallo, la feligresía de San Pedro de Leiroa, cerca de Canderrey ${ }^{18}$.

Sin embargo los descendientes de Pay Mendez de Canderrey no pudieron consolidar la posesión de este territorio por falta de sucesión masculina. Sus hijas vendieron en una fecha algo anterior al año 1333 la fortaleza de Abavides y sus derechos a Ruy Páez de Biedma, un personaje en pleno ascenso social en la comarca; por lo que parece, no hubo descendencia masculina del teniente regio.

Efectivamente, desde 1333 los Biedma aparecen como señores de las tierras de Canderrey a lo largo de varias generaciones que se prolongan hasta el siglo $\mathrm{XV}^{19}$. Durante más de un siglo los miembros del nuevo linaje obtendrán nuevas mercedes reales en territorios próximos a Canderrey, de modo que la fortaleza pierde relevancia al perderse dentro de un conjunto territorial mayor, en el que tiende a tener mayor importancia la posesión de Monterrey, en plena expansión.

Todavía a fines del siglo XVIII la tierra de Canderrey estaba integrada dentro del término de Viladerrey: en la descripción que hace González de Ulloa de los estados de Monterrey en 1777 se dice que «Era antiguamente Villa de Rey capital de unos más de cuarenta lugares, que se nombraban los alfóces de Can de Rey ${ }^{20}$.

Pero pasemos a decir algo de la fortaleza de Abavides, también perteneciente en orígen al realengo, que acabó en manos de Pay Méndez de Canderrey. Bajando el puerto de Estivadas en dirección a Xinzo de Limia,

${ }^{18}$ R.A.H., col. Salazar, M-4 (actual 9/811), fol. 135. Reg. CUARTERO Y HUERTA B., Índice, doc. 45.470, p. 370-371. Alfonso XI confirma esta donación en agosto de 1329; R.A.H., col. Salazar, M-4 (actual 9/811), fol. 135. Reg. CUARTERO Y HUERTA, Índice, doc. 45.471 , p. 371.

${ }^{19}$ VÁZQUEZ LÓPEZ, M.J., «El señorío de Monterrei. Los Biedma, los Stúñiga y los Ulloa», Estudios Mindonienses, 13 (1997), pp. 187-308.

${ }^{20}$ GONZÁLEZ DE ULLOA, Decripción de los estados de la Casa de Monterrey, p. 124; el subrayado es del autor.

«CUADERNOS DE ESTUDIOS GALLEGOS», Tomo XLVIII, Fascículo 114, Santiago 2001. 
y continuando por la antigua Vía de la Plata, se encuentra a seis kilómetros la parroquia de Abavides, o San Martiño de Abavides, perteneciente al actual ayuntamiento de Trasmiras, en donde existió una fortaleza hoy desaparecida. Aún era visible en 1777 cuando González de Ulloa hizo la descripción de los estados de Monterrey ${ }^{21}$, pero ya no debía de serlo en 1845, porque el Diccionario de Madoz omite su existencia. Probablemente no fue una fortaleza demasiado importante. Sin embargo las fuentes documentales cubren con seguridad un corto período cronológico que comienza en 1289 y que se prolonga hasta 1333.

La mención la encontramos, como acabamos de ver hace un momento, en la carta de Sancho IV fechada desde Burgos el 8 de marzo de 1289, por la que hace donación del señorío de la fortaleza de San Martiño de Abavides a Pay Mendey de Canderrey ${ }^{22}$, lo cual demuestra que en esa fecha tanto la fortaleza como su señorío ya existían y además pertenecían al realengo.

El destino final de esta fortaleza es semejante a la de Canderrey: acaba pasando al linaje de los Biedma por culpa de la extinción de la varonía de Pay Méndez. El 28 de febrero de 1333, desde Valladolid, Alfonso XI hace donación de Abavides a Ruy Páez de Biedma, merino mayor de las merindades de Galicia y mayordomo mayor de Pedro Fernández de Castro, conde de Lemos ${ }^{23}$.

\section{MONTERREY Y LA PUEBLA DE FLOR DE REY}

Los orígenes más remotos de la fortaleza realenga de Monterrey datan del primer tercio del siglo XIII, al menos de una manera segura, aunque en ocasiones se ha dicho que bien pudo haber con anterioridad algún tipo de fortificación, tal vez por el hecho de que está enclavada en el antiguo castro Baroncelli, Baruncelle o Varoncelli, o simplemente Castro de Verín.

${ }^{21}$ «Hubo aqui fortaleza de la que aun hay vestigios»; GONZÁLEZ DE ULLOA, Decripción de los estados, p. 153.

${ }^{22}$ R.A.H., Col. Salazar y Castro, Índice, M-4 (signatura actual, 9/811), fol. 135v. Reg. CUARTERO Y HUERTA, Índice, $\mathrm{n}^{\circ} 45.472$.

${ }^{23}$ R.A.H., col. Salazar, M-4 (actual 9/811), fol. 135v; Reg. CUARTERO Y HUERTA B., Índice, doc. $\mathrm{n}^{\circ} 45.473$.

«CUADERNOS DE ESTUDIOS GALLEGOS», Tomo XLVIII, Fascículo 114, Santiago 2001. 
Su posición estratégica en el valle del Támega, vía natural de acceso hacia la comarca de Chaves, hacían de este enclave un lugar ideal para situar una fortaleza, a cuyos pies se encuentran las poblaciones de Verín y Pazos.

Fray Benito de la Cueva, en su Historia de los prioratos de Celanova ${ }^{24}$, obra escrita a mediados del XVII, ya advertía que el nombre de Monterrey era relativamente moderno, datable en el primera tercio del siglo XIII. También glosaba con bastante exactitud todos los detalles relacionados con la propiedad del castro, parte integrante del patrimonio de Celanova desde el siglo $\mathrm{X}$, al igual que sus alrededores, todos ellos dentro del territorio de Varonceli. Las colecciones documentales publicadas en estos últimos años corroboran sus asertos.

La documentación gallega de Alfonso VII, por ejemplo, reafirma la propiedad que Celanova tenía sobre Verín y valle de Varoncelli; en carta de 4 de febrero de 1155 el emperador restituye al citado cenobio todas las posesiones que había tenido en tiempos de Alfonso VI (1065-1109) y que había perdido durante el reinado de Urraca (1109-1126), entre las cuales se citan expresamente las iglesias de «...mitra de Varoncelli, Sancte Marie de Berin cum sua uilla de Moxous» ${ }^{25}$, además de otras iglesias en La Limia. El mismo monarca confirma al monasterio de Celanova el lugar de «Moxios» (o Moxous, actual Mixós), en territorio de Varoncellii ${ }^{26}$. No hay todavía referencia una explícita a fortificaciones.

Sin embargo en 1170 Verín ya es cabeza de una circunscripción territorial o mandación, a cuyo frente figura un tal Fernandus Roderici ${ }^{27}$, que aparece como confirmante en una donación del monarca a favor de Urraca González en ese año; señales ciertas de que Monterrey estaba empezando a convertirse en un punto importante de delimitación fronteriza

${ }^{24}$ CUEVA, B. de la, Historia de los monasterios y prioratos anejos a Celanova (ed. de GONZÁLEZ BALASCH, M.T. y FERNANDEZ DE VIANA, J.I.), Granada, 1991.

${ }^{25}$ RECUERO ASTRAY, M., GONZÁLEZ VÁZQUEZ, M. y ROMERO PORTILLA, P., Documentos medievales del Reino de Galicia: Alfonso VII (1116-1157), La Coruña, 1998, doc. 166, p. 178.

${ }^{26}$ Sin lugar ni data, inserto en una confirmación de Alfonso IX; RECUERO ASTRAY, Alfonso VII, doc. 183, p. 199.

${ }^{27}$ Fechado en León, el 30 de junio de 1170; RECUERO ASTRAY, M., ROMERO PORTILLA, P., y RODRIGUEZ PRIETO, M.A., Documentos medievales del Reino de Galicia: Fernando II (1155-1188), La Coruña, 2000, doc. 118, p. 147. 
ante el recién nacido Portugal, ya que es esa época seguía habiendo tenentes por el rey en La Limia.

Durante el reinado de Alfonso IX de León (1188-1230) se realizaron en el castro los primeros intentos de poblamiento organizado, lo que provocó la protesta del monasterio de Celanova. El monarca decidió finalmente ceder ante las demandas del cenobio y ordenó, el 3 de marzo de 1223 (desde Allariz), deshacer todo lo que se había construido en el castro hasta ese momento:

«... ego Adephonsus, Dei gratia rex Legionis et Gallecie et Asturiarum, intuiti pietatis et misericordie et ad preces abbatis et conuentus monasterii Cellenoue, quia intellexi et perpendi maximum dampnum et graue detrimentum inminere ipsi monasterio Cellenoue per populationem illam que fiebat in Castro de Berim, in terra de Barunceli, mando et precipio ipsam populacionem statim destrui, et concedo e confirmo quod numquam ego nec aliquis de successoribus meis faciat ibi populacionem fieri ${ }^{28}$.

Entre los confirmantes de esta orden regia figura "Domno Martino Sancii, signifero domini regis, tenente Limiam et Toronium et Sarriam et Baruncelim», lo cual demuestra que la tenencia de Baronceli seguía siendo, a pesar del desmantelamiento, una circunscripción regia importante. Julio Gonzále ${ }^{29}$ enmarca este intento fallido de poblar el castro de Verín dentro del conjunto de repoblaciones del sur de Galicia y la frontera portuguesa, como Salvatierra (1228), Burgo de Caldelas (1228), Mellid (1213), o incluso Monforte de Lemos (1199).

El abad de Celanova se tomó la molestia de acudir a la curia romana para reafirmar sus derechos y así obtuvo de Honorio III, el 10 de abril de 1225 , una sanción canónica que ratificaba las disposiciones de Alfonso

${ }^{28}$ GONZÁLEZ, J., Alfonso IX, vol. II, doc. 429, pp. 544-545. SÁNCHEZ BELDA, Documentos reales de la Edad Media referentes a Galicia, Madrid, 1953, $\mathrm{n}^{\circ}$ 527; VAQUERO DÍAZ, M.B., Colección Diplomática do mosteiro benedictino de San Salvador de Celanova (Ourense) (séculos XIII, XIV e XV), Tesis doctoral, Univ. de Vigo, 2001, doc. 9 y confirmación en doc. 15. Citado también por CUEVA, Historia de los monasterios, cap. 15 , p. 127 , nota 65 .

${ }^{29}$ GONZÁLEZ, J., Alfonso IX, vol. I, pp. 248-251.

«CUADERNOS DE ESTUDIOS GALLEGOS», Tomo XLVIII, Fascículo 114, Santiago 2001. 
IX sobre el desmantelamiento del castro ya que pertenecía al monasterio $^{30}$. Alfonso IX reiteró otra vez al monasterio de Celanova en marzo de 1226 la promesa de que el castro de Verín no sería repoblado ${ }^{31}$. A lo largo de las décadas siguientes los abades de Celanova obtuvieron confirmaciones de una y otra carta: de la del rey, que prohibía la población de Verín, hubo confirmaciones de Fernando III en $1232^{32}$, de Alfonso X en $1255^{33}$, de Fernando IV en $1300^{34}$ y de Alfonso XI en $1316^{35}$. De la Curia romana se obtuvo confirmación del Papa Clemente IV en $1266^{36}$.

Pero a pesar de todas estas confirmaciones hubo poblamiento organizado en el castro durante la segunda mitad del siglo XIII y, además, por iniciativa personal de Alfonso X. Lo demuestra una carta del rey Sabio, fechada en 1274, en la que hace donación al monasterio de Celanova de la mitad de la iglesia de Monterrey que él mismo había mandado levantar:

«Les do la meatade de la egleia que agora nuevamente mande fazer en la mi Prueba de Monte de [Rey]» ${ }^{37}$.

Efectivamente, Monterrey se estaba convirtiendo en puebla de realengo a pesar del disgusto del monasterio de Celanova, que ya poseía Verín y las tierras comarcanas del valle de Varonceli. Fray Benito de la Cueva

${ }^{30}$ GONZÁLEZ, J., Alfonso IX, I, p. 249. VAQUERO DÍAZ, Colección Diplomática, doc. 11 .

${ }^{31}$ GONZÁLEZ, J., Alfonso IX, II, doc. 487, p. 591.

${ }^{32}$ Fechada en Orense, el de abril de 1232; VAQUERO DÍAZ, Colección Diplomática, doc. 35 .

${ }^{33}$ Fechada en Valladolid, a 18 de septiembre de 1255; VAQUERO DÍAZ, Colección Diplomática, doc. 57.

${ }^{34}$ Fechada en 25 de marzo de 1330; reg. SÁNCHEZ BELDA, Documentos reales, $\mathrm{n}^{\circ}$ 796; BALLESTEROS, A., Alfonso X el Sabio, reed. Barcelona, 1984, $n^{\circ}$ 933; CUEVA, Historia de los monasterios y prioratos, p. 128, nota 67.

${ }^{35}$ Fechada en Toro el 18 de agosto de 1316; VAQUERO DÍAZ, Colección Diplomática, doc. 103 .

${ }^{36}$ Fechada en Viterbo el 27 de septiembre de 1266; VAQUERO DÍAZ, Colección Diplomática, doc. 61. Clemnete IV ordena que no se reedifique ninguna iglesia en el castro de Verín y que ningún presbítero celebre allí la misa, caso de haber alguna iglesia.

${ }^{37}$ Fechada en Palencia el 16 de abril de 1274; Reg. SÁNCHEZ BELDA, Documentos reales, $\mathrm{n}^{\circ} 796$; BALLESTEROS, Alfonso $X, \mathrm{n}^{\circ}$ 933; ed. VAQUERO DÍAZ, Colección Diplomática, doc. 67. Carta confirmada por Sancho IV, desde Burgos, el 10 de mayo de 1291; VAQUERO DÍAZ, Colección Diplomática, doc. 83. 
opina, apoyándose en este texto, que en este momento ya se habrían iniciado las labores de construcción del castillo; pero en realidad el documento en cuestión sólo habla de la iglesia del castro, no de la fortaleza ${ }^{38}$. Sin embargo parece lógico suponer que la repoblación del castro tendría sentido precisamente por su valor defensivo y que bien pudo haber algún tipo de fortificación.

Además de Alfonso X, el responsable indirecto de la fundación de la «puebla» de Monterrey fue el adelantado de Galicia Andrés Fernández de Castro, que tenía este cargo al menos desde $1265^{39}$, aunque su nombre figura entre los conspiradores que se coaligaron contra el rey en 1273, entre otras cosas, por haber ordenado la fundación de pueblos nuevos en León y Galicia ${ }^{40}$. Consta claramente que Estéban Fernández de Castro deslindó los terrenos de la nueva puebla con los de Verín: así lo prueba la documentación de un pleito que se desarrolló, durante el reinado de Sancho IV, entre el monasterio de Celanova, por una parte, y el concejo de Moneterrey, por otra. El rey Bravo ordenó en mayo de 1293, a la vista de las pruebas, que se hiciera un nuevo deslinde; entre esas pruebas, el abad de Celanova afirma que Estéban Fernández puso marcas y divisiones entre Monterrey y los cotos pertenecientes a Celanova, cumpliendo instrucciones de Alfonso $\mathrm{X}^{41}$.

Sancho IV impulsó la repoblación del castro a través de su agente en la zona, Pay Méndez de Canderrey, que tuvo la tenencia de las pueblas de Monterrey, Viladerrrey y «Friol de Rey» (sic, por Flor de Rey) a fines del siglo XIII y comienzos del XIV. En estos años se impone definitivamente el nombre de Monterrey frente a la antigua denominación de Varoncelli.

Pay Méndez de Canderrey fue el responsable de las obras de construcción del cercano castillo de Lobarzana, situado cerca de Villaza, probablemente en el castro que hoy se llama de Lobarzán. Este teniente obligó a los vasallos de la zona, sujetos a Celanova, a que trabajasen en las obras

\footnotetext{
${ }^{38}$ CUEVA, Historia de los monasterios y prioratos, p. 128.

${ }^{39}$ PARDO DE GUEVARA, E., Los señores de Galicia. Tenentes y condes de Lemos en la Edad Media, I, La Coruña, 2000, p. 113. Un perfil biográfico en pp. 113-122.

${ }^{40}$ PARDO DE GUEVARA, E., Los señores de Galicia, I, p. 116.

${ }^{41}$ 1293, mayo, 14; Valladolid. Sancho IV manda demarcar los términos de Monterrey y Verín para acabar el pleito que enfrenta al monasterio de Celanova con el concejo de Monterrey. Ed. VAQUERO DÍAZ, Colección Diplomática de Celanova, doc. $\mathrm{n}^{\circ} 85$.
} 
de ese castillo, y por eso el cenobio protestó ante el rey. Finalmente, en 1292, Sancho IV ordenó a su oficial que no volviera a molestar a los vecinos de los cotos de Pazos, Verín y Moixós, argumentando que nunca habían estado sujetos a tales obligaciones ${ }^{42}$. ¿Se estaban haciendo también obras en el castillo de Monterrey? No lo sabemos por ahora, pero no sería de extrañar que las obras de construcción del cercano castillo de Lobarzana obedeciesen a las dificultades puestas una y otra vez por los monjes de Celanova sobre el castro de Verín.

Las cuentas de Sancho IV de los años 1290 y 1292 revelan que el rey tenía en «La puebla de Monterrey, con val de Varonçella (o Baronçelle)» 6.000 maravedíes anuales, que estaban puestos a favor de Juan Fernández de Limia, uno de los grandes señores de Galicia. Las cuentas no hablan para nada de posibles rentas de un castillo en Monterrey, a diferencia de lo que sucedía con el cercano Canderrey, que estaba en manos de Pay Méndez de Canderrey, y que generaba una renta propia del castillo. Pero durante su reinado, siendo Pay Gómes adelantado mayor de Galicia, prosiguió el pleito entre Celanova y la puebla de Monterrey sobre el deslinde de términos que ya hemos citado, aunque la documentación no aclara la posible existencia de una fortaleza ${ }^{43}$.

Queda por descifrar el enigma de la puebla de Flor de Rey, en el término del actual Vilardevós, donde se encuentra un castro al que siempre se le ha supuesto una historia prerromana; la referencia de 1292 indica claramente que había allí una «puebla» encomendada a Pay Méndez, pero no se dice nada de un castillo ${ }^{44}$. Desgraciadamente este topónimo no aparece entre los lugares entregados o donados a los Biedma en el primer tercio del siglo XIV, bien por donación regia, bien por las compras que se hicieron a las hijas de Pay Méndez: ¿estamos tal vez ante un caso de repoblación fracasada? La incógnita sigue a la espera de una respuesta.

\footnotetext{
${ }^{42}$ Carta fechada en Naves Frías el 8 de mayo de 1292; ed. VAQUERO DÍAZ, Colección Diplomática de Celanova, doc. 84.

${ }^{43}$ 1293, julio, 19; Monterrey. Pay Gómez, adelantado del rey en Galicia, con consentimiento del monasterio de Celanova y del concello de Monterrey, elige unos hombres buenos para deslindar los términos; ed. VAQUERO DÍAZ, Colección Diplomática de Celanova, doc. $\mathrm{n}^{\circ} 86$.

${ }^{44}$ Ver nota 41.
} 